\title{
Myasthenic Crisis Complicated with Myxedema, Positive for Both Anti-acetylcholine Receptor and Anti-muscle-specific Tyrosine Kinase Antibodies
}

\author{
Kazuhiro Horiuchi ${ }^{1}$, Azusa Nagai ${ }^{1}$, Masahiro Wakita ${ }^{1}$, Shotaro Ito ${ }^{2}$, \\ Kei Takamura ${ }^{2}$ and Hideki Houzen ${ }^{1}$
}

\begin{abstract}
:
We herein report the case of myasthenic crisis occurring in a 51-year-old man. He had experienced ptosis, increased body weight with edema, and fatigue with dyspnea. He presented at our emergency department with disturbed consciousness. He was originally diagnosed with myxedema coma, and he required artificial respiration. Because his weakness persisted and he was positive for anti-acetylcholine receptor antibodies and anti-muscle-specific tyrosine kinase antibodies, we diagnosed myasthenic crisis after various examinations. His clinical response to treatment was good and he was discharged in an ambulatory status 3 months after admission. This case demonstrates that myasthenic crisis may occur in association with myxedema.
\end{abstract}

Key words: myasthenia gravis, myxedema coma, myasthenic crisis, anti-acetylcholine receptor antibody, antimuscle-specific tyrosine kinase antibody

(Intern Med 57: 265-268, 2018)

(DOI: 10.2169/internalmedicine.9291-17)

\section{Introduction}

Myasthenia gravis (MG) is a chronic autoimmune neuromuscular disease characterized by insufficient neuromuscular transmission leading to progressive muscle weakness. It is frequently accompanied by autoimmune thyroid diseases, which may influence its course $(1,2)$. Myasthenic crisis is characterized by weakness of the respiratory and bulbar muscles, leading to acute respiratory failure requiring mechanical ventilatory support. Myxedema coma, a well-known life-threatening condition, is an emergency presentation of hypothyroidism (3). We herein report a case of a myasthenic crisis complicated with myxedema.

\section{Case Report}

We encountered a case of a myasthenic crisis in a 51year-old man. The patient had a history of hypothyroidism identified over 5 years prior to this presentation, which had not been treated because it was asymptomatic. He had also undergone meningioma surgery 5 years previously. His family history was unremarkable. He had experienced bilateral ptosis for 10 months, his body weight had increased with edema (20 kg in 3 months), and he had suffered from fatigue with dyspnea for 1 month before admission. He presented to our emergency department with a disturbed consciousness.

On examination, he appeared ill and drowsy, with a pulse rate of 50 beats $/ \mathrm{min}$, blood pressure of $220 / 92 \mathrm{mmHg}$, respiratory rate of 10 breaths/min, oxygen saturation of $75 \%$, and temperature of $37.0^{\circ} \mathrm{C}$. His height was $169.5 \mathrm{~cm}$, weight was $110 \mathrm{~kg}$, and body mass index (BMI) was $38.29 \mathrm{~kg} / \mathrm{m}^{2}$. His breath sounds were clear on chest auscultation. Wholebody edema was prominent.

On neurological examination, he was drowsy with a Glasgow Coma Scale score of 12 (E2V4M6), bilateral ptosis, left eye movement restriction in all directions, bilateral facial weakness, difficulty in swallowing, dysphagia, difficulty of tongue movement, and general weakness [cervical flexor muscle Manual Muscle Test (MMT) was 2/5, limb proximal muscle MMT was 2/5, and limb distal muscle MMT was

${ }^{1}$ Department of Neurology, Obihiro Kosei General Hospital, Japan and ${ }^{2}$ First Department of Medicine, Obihiro Kosei General Hospital, Japan Received: April 1, 2017; Accepted: May 16, 2017; Advance Publication by J-STAGE: November 1, 2017 Correspondence to Dr. Kazuhiro Horiuchi, horiuchi-kazuhiro@hotmail.co.jp 


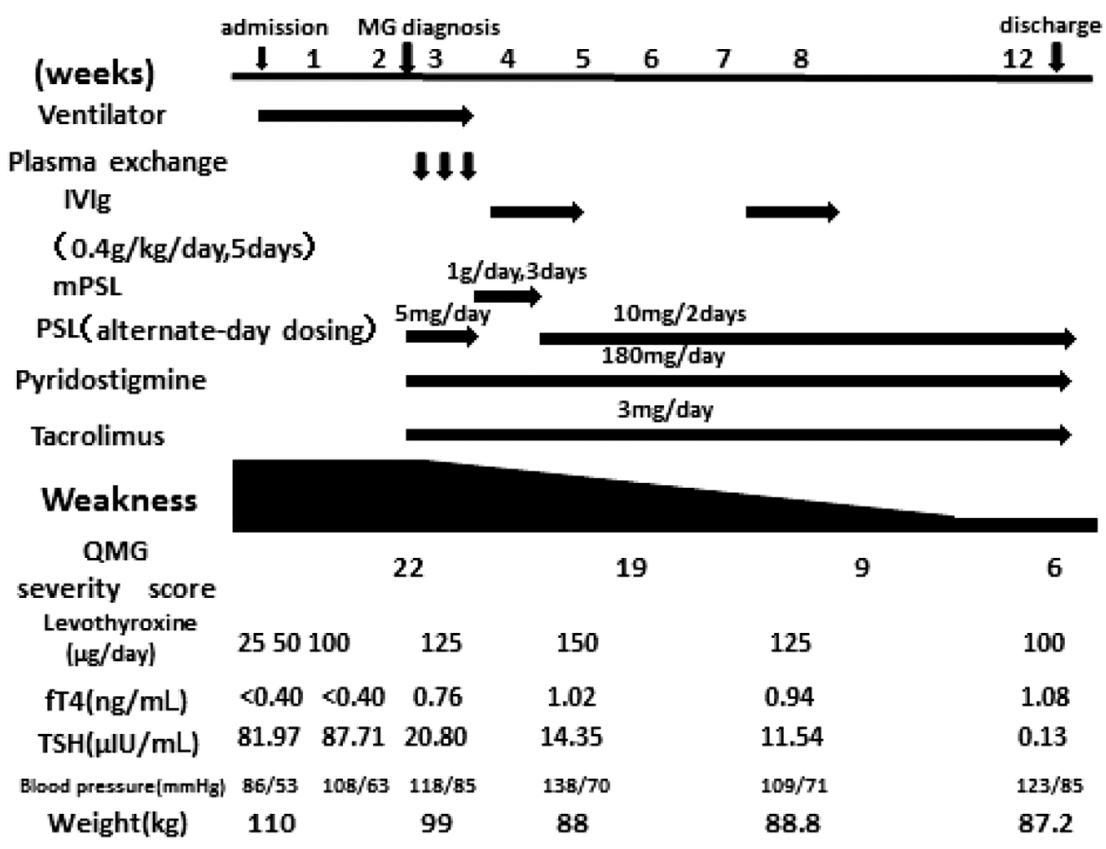

Figure. The patient's clinical course. Myasthenia gravis (MG) symptoms (weakness) were resolved by plasma exchange, intravenous immunoglobulin (IVIg), methylprednisolone (mPSL), prednisolone (PSL), pyridostigmine, and tacrolimus. The quantitative myasthenia gravis (QMG) severity score improved from 22 to 6 . His hypothyroidism gradually improved, body weight declined, and blood pressure stabilized due to the administration of levothyroxine.

3/5). Initial laboratory studies showed the following: white

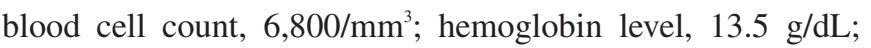
platelet count, $166 \times 10^{3}$; C-reactive protein value, $0.07 \mathrm{mg}$ / $\mathrm{dL}$; aspartate aminotransferase, 47 international units (IU)/L; alanine aminotransferase, $33 \mathrm{IU} / \mathrm{L}$; blood urea nitrogen, 17.0 $\mathrm{mg} / \mathrm{dL}$; creatinine, $0.87 \mathrm{mg} / \mathrm{dL}$; sodium, $143 \mathrm{mEq} / \mathrm{L}$; potassium, $4.2 \mathrm{mEq} / \mathrm{L}$; and chloride, $96 \mathrm{mEq} / \mathrm{L}$. His free $\mathrm{T} 3$ was $1.05 \mathrm{pg} / \mathrm{mL}$ (normal range, $1.71-3.71 \mathrm{pg} / \mathrm{mL}$ ) and free $\mathrm{T} 4$ was under $0.4 \mathrm{ng} / \mathrm{dL}$ (normal range, 0.7-1.48 ng/dL). The level of thyroid-stimulating hormone was elevated to 81.97 $\mu \mathrm{U} / \mathrm{L}$ (normal range, 0.35-4.94 $\mu \mathrm{U} / \mathrm{L}$ ), and the levels of antithyroid peroxidase and anti-thyroglobulin antibodies exceeded 4,000 IU/mL (normal range, <27.9 IU/mL) and 600 $\mathrm{IU} / \mathrm{mL}$ (normal range, $<15.9 \mathrm{IU} / \mathrm{mL}$ ), respectively. Arterial blood gas testing, conducted while the patient was breathing oxygen at $1 \mathrm{~L} / \mathrm{min}$ via a nasal cannula, revealed a $\mathrm{pH}$ of 7.32, partial pressure of $\mathrm{CO}_{2} 86.8 \mathrm{mmHg}$, partial pressure of $\mathrm{O}_{2} 53.3 \mathrm{mmHg}$, and $\mathrm{HCO}_{3}{ }^{-}$of $43.7 \mathrm{mmol} / \mathrm{L}$. He was diagnosed as having $\mathrm{CO}_{2}$ narcosis due to myxedema coma arising from Hashimoto's thyroiditis. Treatment with levothyroxine and corticosteroids was initiated. He required artificial respiration.

The patient's clinical course is outlined in Figure. We stopped corticosteroids because his adrenal function was normal. By regulating the respirator and gradually increasing the levothyroxine, he regained a clear consciousness. However, withdrawal from the respirator was impossible even 2 weeks after admission because of general weakness. He was referred to the Neurology Department.

The Tensilon test was positive, indicating MG, and a neu- rophysiological examination with repetitive nerve stimulation of the left accessory nerve was also positive. His antiacetylcholine receptor (AChR) antibody level was $80 \mathrm{nmol} / \mathrm{L}$ (normal range, $<0.2 \mathrm{nmol} / \mathrm{L}$ ) and his anti-muscle-specific tyrosine kinase (MuSK) antibody level was $0.15 \mathrm{nmol} / \mathrm{L}$ (normal range, $<0.02 \mathrm{nmol} / \mathrm{L}$ ); both were positive. Thoracic computed tomography (CT) and magnetic resonance imaging (MRI) showed residual thymic tissue in the anterior mediastinum. We diagnosed myasthenic crisis, with a Myasthenia Gravis Foundation of America clinical classification of $\mathrm{V}$ and a quantitative MG (QMG) severity score of 22 . He was started on treatment with pyridostigmine (180 mg/day), oral prednisolone (10 mg, alternate days), plasma exchange (5\% albumin, $40 \mathrm{~mL} / \mathrm{kg}$ ), and tacrolimus (3 mg/day). Plasma exchange was stopped after three procedures because of bleeding from the insertion site of the catheter. After the plasma exchanges, we treated him with methylprednisolone pulse therapy ( $1 \mathrm{~g} /$ day) for 3 days and intravenous immunoglobulin (IVIg; $0.4 \mathrm{mg} / \mathrm{kg} / \mathrm{day}$ ) for 5 days. After treatment, the patient showed a good clinical response, with a gradual improvement in his ptosis, general fatigue, and weakness, and 10 days after this treatment, we were able to take him off the ventilator. Three months after admission, he was able to walk without assistance and was discharged from our hospital. His QMG severity score improved from 22 to 6 . Because he was positive for anti-AChR antibodies, we considered performing thymectomy; however, this was not performed as the patient refused the procedure. 


\section{Discussion}

MG is frequently accompanied by autoimmune thyroid diseases. In one study, these were diagnosed in $26.8 \%$ of 343 consecutive MG patients, including $4.4 \%$ with Graves' disease, $9.0 \%$ with Hashimoto's thyroiditis, and $13.4 \%$ only with antithyroid antibodies (2). In a Japanese study, $11.9 \%$ of MG patients had Graves' disease or Hashimoto's thyroiditis (1). In recent years, there has been no reported case of myasthenic crisis complicated with myxedema. However, the risk of myasthenic crisis and the results of treatment did not differ between the patients with and without autoimmune thyroid diseases $(2,3)$.

In the present case, hypothyroidism and MG together resulted in the onset of myxedema coma and myasthenic crisis. Hypothyroidism was asymptomatic for approximately 5 years; however, he was aware of the increase in his body weight by $20 \mathrm{~kg}$ in 3 months along with edema.

There were reports of hypercapnia, severe hypothermia, and cardiac involvement with the development of multiorgan dysfunction in patients with myxoedema coma that mentioned hypoxaemia in $80 \%$ and hypercapnia in $54 \%$ of the patients. One way to explain the respiratory failure observed in this case could be the decreased sensitivity of the central nervous system to hypoxia and hypercapnia $(4,5)$.

On admission, we were not able to diagnose the patient with myasthenic crisis. We considered severe hypothyroidism had likely induced consciousness disturbance with $\mathrm{CO}_{2}$ narcosis and ptosis with edema.

In myasthenic crisis, respiratory muscle fatigue deteriorates, leading to respiratory failure and $\mathrm{CO}_{2}$ narcosis (6). Although myxedema coma and myasthenic crisis both cause consciousness disturbance with $\mathrm{CO}_{2}$ narcosis $(5,6)$, we thought that the patient's prominent edema (myxedema) with weight gain due to untreated hypothyroidism had caused respiratory muscle fatigue with $\mathrm{MG}$, finally resulting in myasthenic crisis.

Because MG is often associated with hypothyroidism, clinicians should perform examinations and diagnose it immediately when myxedema coma and myasthenic crisis is considered to be possible based on the medical history and physical findings. In addition, treatment for both hypothyroidism and MG should be provided promptly.

The anti-MuSK antibody is present in $10-70 \%$ of all MG patients without anti-AChR antibodies and this MG subtype is clinically more severe than others, with the involvement of facial, bulbar, and upper body muscles, and sometimes with muscle atrophy (7). The effect of an edrophonium injection (the Tensilon test) is equivocal or negative in some patients, and the response to oral pyridostigmine can be even more unsatisfactory, ranging from some mild benefit to overt intolerance. The course of the disease is often characterized by periodic exacerbation phases that require hospitalization and even assisted ventilation; plasma exchange can result in a marked improvement in these cases (7). In gen- eral, MuSK antibody is measured when AChR antibody is negative. However, in this case wherein bulbar palsy and respiratory failure occurred rapidly, we considered MuSK antibody positive MG. If MuSk antibody was positive, then plasma exchange was more desirable than immunoadsorption in the treatment of myasthenic crisis $(8,9)$. Additionally, if only AchR antibody was positive, then immunoadsorption was considered to be indicated. Therefore, both antibodies were measured. The coexistence of anti-AChR and MuSK antibodies has been reported in only a few cases (10). These patients were anti-AChR antibody-positive at disease onset but became anti-MuSK antibody-positive after thymectomy (11-13). D-penicillamine can induce both anti-AChR- and MuSK antibody-positive MG, a rare phenomenon which is reversible after the discontinuation of the treatment (14). In the present case, the effect of the edrophonium injection was remarkable, and the patient had no problem with the tolerance of oral pyridostigmine; however, respiratory failure rapidly appeared due to respiratory muscle fatigue, which was consistent with the characteristics of anti-MuSK antibody-positive MG.

The patient showed no thymoma on CT and MRI. In general, late onset MG without thymoma is not indicated for thymectomy. However, a randomized trial by Wolfe et al. states that thymectomy improved the clinical outcomes over a 3-year period in 18-65 years old patients with nonthymomatous AChR antibody positive MG (15). In anti-MuSK antibody-positive patients, the thymus was normal for their age or was atrophied, and no benefit from thymectomy was observed (7). In cases with the coexistence of anti-AChR and anti-MuSK antibodies, it is not clear whether or not to perform a thymectomy (13). In the present case, we considered thymectomy after careful consideration of the exacerbations of the patient's symptoms. However, the patient refused, and thymectomy was therefore not performed.

\section{Conclusion}

In summary, we herein reported a case of a myasthenic crisis complicated with myxedema. MG is frequently accompanied by autoimmune thyroid diseases, and clinicians should remain aware of the possibility of cases of myxedema coma with $\mathrm{CO}_{2}$ narcosis due to myasthenic crisis.

\section{The authors state that they have no Conflict of Interest (COI).}

\section{Acknowledgement}

We would like to thank the staff of the medical unit for their valuable help in the management of this patient.

\section{References}

1. Kanazawa M, Shimohata T, Tanaka K, Nishizawa M. Clinical features of patients with myasthenia gravis associated with autoimmune diseases. Eur J Neurol 14: 1403-1404, 2007.

2. Kubiszewska J, Szyluk B, Szczudlik P, et al. Prevalence and impact of autoimmune thyroid disease on myasthenia gravis course. 
Brain Behav 6: e00537, 2016.

3. Klubo-Gwiezdzinska J, Wartofsky L. Thyroid emergencies. Med Clin North Am 96: 385-403, 2012.

4. Salomo LH, Laursen AH, Reiter N, Feldt-Rasmussen U. Myxoedema coma: an almost forgotten, yet still existing cause of multiorgan failure. BMJ Case Reports: 2014.

5. Lacomis D. Myasthenic crisis. Neurocrit Care 3: 189-194, 2005.

6. Wall CR. Myxedema coma: diagnosis and treatment. Am Fam Physician 62: 2485-2490, 2000.

7. Yeh JH, Chen WH, Chiu HC, Bai CH. MuSK antibody clearance during serial sessions of plasmapheresis for myasthenia gravis. J Neurol Sci 263: 191-193, 2007.

8. Suzuki H, Motomura M, Yoshimura T, et al. A case of myasthenia gravis with anti-MuSK antibodies showing a dramatic improvement with plasma exchange. Rinsho Shinkeigaku 45: 510-513, 2005 (in Japanese, Abstract in English).

9. Evoli A, Tonali PA, Padua L, et al. Clinical correlates with antiMuSK antibodies in generalized seronegative myasthenia gravis. Brain 126: 2304-2311, 2003.

10. Fernández JA, Fernández-Valiñas A, Hernández D, Orozco J, Lugo A. Myasthenic crisis in an elderly patient with positive antibodies against acetylcholine and anti-MuSK, successfully treated with noninvasive mechanical ventilation. Case Rep Crit Care 2015: 624718, 2015.

11. Saulat B, Maertens P, Hamilton WJ, Bassam BA. Anti-musk antibody after thymectomy in a previously seropositive myasthenic child. Neurology 69: 803-804, 2007.

12. Kostera-Pruszczyk A, Kwiecinski H. Juvenile seropositive myasthenia gravis with anti-MuSK antibody after thymectomy. J Neurol 256: 1780-1781, 2009.

13. Rajakulendran S, Viegas S, Spillane J, Howard RS. Clinically biphasic myasthenia gravis with both AChR and MuSK antibodies. J Neurol 259: 2736-2739, 2012.

14. Poulas K, Koutsouraki E, Kordas G, Kokla A, Tzartos SJ. AntiMuSK- and anti-AChR-positive myasthenia gravis induced by dpenicillamine. J Neuroimmunol 250: 94-98, 2012.

15. Wolfe GI, Kaminski HJ, Aban IB, et al. Randomized trial of thymectomy in myasthenia gravis. N Engl J Med 375: 511-522, 2016.

The Internal Medicine is an Open Access article distributed under the Creative Commons Attribution-NonCommercial-NoDerivatives 4.0 International License. To view the details of this license, please visit (https://creativecommons.org/licenses/ by-nc-nd/4.0/).

(C) 2018 The Japanese Society of Internal Medicine Intern Med 57: 265-268, 2018 